\title{
Trivium
}

Revue franco-allemande de sciences humaines et sociales - Deutsch-französische Zeitschrift für Geistesund Sozialwissenschaften

$3 \mid 2009$

Droits subjectifs et droits de l'homme

\section{Die Interpretation der Menschenrechte. Politische und theoretische Herausforderungen im Spektrum der französischen Diskussion}

\section{Catherine Colliot-Thélène}

Traducteur : Achim Russer

\section{OpenEdition}

Journals

Édition électronique

URL : http://journals.openedition.org/trivium/3306

DOI : $10.4000 /$ trivium.3306

ISSN : 1963-1820

Éditeur

Les éditions de la Maison des sciences de l'Homme

Référence électronique

Catherine Colliot-Thélène, «Die Interpretation der Menschenrechte. Politische und theoretische Herausforderungen im Spektrum der französischen Diskussion », Trivium [En ligne], 3| 2009, mis en ligne le 15 avril 2009, consulté le 22 septembre 2020. URL : http://journals.openedition.org/trivium/ 3306 ; DOI : https://doi.org/10.4000/trivium.3306

Ce document a été généré automatiquement le 22 septembre 2020

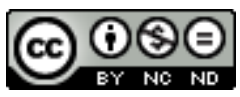

Les contenus des la revue Trivium sont mis à disposition selon les termes de la Licence Creative Commons Attribution - Pas d'Utilisation Commerciale - Pas de Modification 4.0 International. 


\title{
Die Interpretation der Menschenrechte. Politische und theoretische Herausforderungen im Spektrum der französischen Diskussion
}

\author{
Catherine Colliot-Thélène
}

Traduction : Achim Russer

Nachdem der Begriff der Menschenrechte über lange Zeit hinweg in der französischsprachigen Literatur sei es vernachlässigt, sei es durch die herrschenden theoretischen Diskurse unter Verdacht gestellt worden war, haben sich die einschlägigen Veröffentlichungen seit den achtziger Jahren geradezu gehäuft. Auf philosophischer Ebene hatten die Kritik des Subjekts und der Metaphysik der Subjektivität, in der Heideggers Schüler sich zusammenfanden, ein bestimmter Marxismus und unterschiedliche Varianten jener Strömung, die als Strukturalismus bezeichnet zu werden pflegt, vereint darauf hingewirkt, die theoretische Stringenz der Idee der Menschenrechte in Zweifel zu ziehen. Hinzu kamen auf Seiten linker Denker das Misstrauen gegenüber dem Recht im Allgemeinen, dessen wesentliche Funktion viele mit Marx als Dienst an einem Staat betrachteten, der seinerseits im Dienst der herrschenden Klasse steht, sowie die extreme politische Polarisierung der sechziger und siebziger Jahre, neben der die Menschenrechte mit ihrer Universalität und folglich Neutralität gegenüber politischen und sozialen Parteiungen als politisch irrelevant galten. Auf juristischer Seite schließlich trug ein weiterer Faktor zur Diskreditierung der Menschenrechte bei, nämlich die strenge Kritik, der der Rechtshistoriker Michel Villey in seinen Vorlesungen und Schriften den Begriff des subjektiven Rechts unterzog. Gestützt auf eine beeindruckende Gelehrsamkeit und unter virtuosem Einsatz seiner Talente als Polemiker widmete Villey der Kritik der "Menschenrechtsideologie« eine weit über den Kreis der Juristen und Rechtshistoriker hinaus beachtete Monographie. ${ }^{1}$ Noch in einer neueren Veröffentlichung ${ }^{2}$ zitiert der 
Philosoph Vincent Descombes die lapidare Formel, in die Villey seine Grundthese fasste - "Das Recht kennt keine Subjekte, es kennt nur Rechtsträger« -, die jener sich im Rahmen seiner wittgensteinisch inspirierten Kritik der verschiedenen Abwandlungen des Subjektbegriffs zu Eigen macht. Ungeachtet des unterschiedlichen theoretischen Rahmens erhebt Descombes denselben Anspruch wie Villey, den "Streit um die subjektiven Rechte« durch Verzicht auf den Begriff Rechtssubjekt zu lösen.

Wie kommt es, dass die Thematik der Menschenrechte trotz dieser starken theoretischen und politischen Widerstände seit der Wende der siebziger und achtziger Jahre eine in Artikeln und Monographien bis heute anhaltende Hochkonjunktur erlebt? Gewiss, die Kritik ist nicht völlig verstummt. Schon 1980 wurde sie von Marcel Gauchet erneuert, der gegenüber denen, die in den Menschenrechten einen Ersatz für einen totgelaufenen politischen Diskurs der Linken suchten, die Auffassung vertrat, dass »die Menschenrechte keine Politik sind «. ${ }^{3}$ Einige Jahre später erinnerte Bertrand Binoche an die diversen Spielarten der Kritik der Menschenrechte seit der Epoche der Französischen und der Amerikanischen Revolution. ${ }^{4}$ Und Vincent Descombes greift, wie gesagt, diesen Streit zu Beginn des neuen Jahrhunderts auf. Man kann sogar sagen, dass diese Kritik in den letzten dreißig Jahren expliziter zum Ausdruck kam als in vorangegangenen Jahrzehnten, in denen die Idee der Menschenrechte allgemein derart obsolet erschien, dass selten die Notwendigkeit empfunden wurde, sich dabei aufzuhalten. Aber wenn diese Kritik expliziter wurde, so in Reaktion auf eine neue politisch-theoretische Konjunktur, in der die Menschenrechte wieder den Status eines politischen Gegenstands erhielten. Wenn daher die jüngere Kritik dieses Begriffs sich bei Gelegenheit durchaus auch aus dem Arsenal der von der älteren Kritik gelieferten Argumente bedient, ist sie dennoch neu und ihre Argumentation zumindest teilweise originell, wie es ja auch der Kontext ist, in dem die Menschenrechte wieder zum Gegenstand politischer Debatten und Kämpfe aufgestiegen sind.

Wenn seit dem Ende der siebziger und deutlicher noch seit dem Beginn der achtziger Jahre in der ideologischen und politischen Geschichte der Menschenrechte in Frankreich ein Wendepunkt eintrat, der sich nach Jahrzehnten der Gleichgültigkeit oder Verachtung in einer Aufwertung ihrer politischen Bedeutung niederschlug, so haben sich im Lauf der folgenden Jahre auch die Faktoren des politisch-sozialen Kontextes geändert, die seit diesem Wendepunkt für ein kontinuierliches Interesse an den Menschenrechten sorgen. Neue, aus der politischen Aktualität heraus entstandene Befürchtungen traten an die Stelle derer, die in den achtziger Jahren das neue Interesse ausgelöst hatten, oder gesellten sich ihnen hinzu. Wir werden uns hier mit einer raschen Aufzählung der Faktoren begnügen, die diesen Kontext und seine Entwicklung gemeinsam oder nacheinander bestimmten.

Zunächst einmal war dies am Ende der siebziger Jahre die Unterstützung der Dissidenten des Sowjetblocks und die Kritik der totalitären Regime. ${ }^{5}$ Zehn Jahre später gab die Zweihundertjahrfeier der Französischen Revolution, die der historische Zufall mit dem Zusammenbruch des Sowjetregimes zusammenfallen ließ, Anlass zu einem neuen Studium der Quellen. Dem entsprach direkt ${ }^{6}$ oder indirekt ${ }^{7}$ die Veröffentlichung grundlegender Texte und Kommentare. Die Ereignisse des Jahres 1989 lösten weltweit politische Krisen aus, in denen die klassischen Deutungsschemata (der Rechten wie der Linken) mehr oder weniger vollständig außer Kurs gesetzt und in deren Verlauf Massaker begangen wurden (das Auseinanderbrechen Jugoslawiens und seine Folgen). Das war die Stunde des »Humanitären«, das sich nur auf die Menschenrechte berufen 
konnte, die es zugleich in einer Logik des Mitleidens entpolitisierte - einer Logik, die vor allem dazu diente, die Unfähigkeit des Politischen zu bemänteln. ${ }^{8}$ Parallel dazu erweiterte die Frage der Immigration, in Frankreich von parteitaktischen Erwägungen zu einem zentralen Wahlkampfthema erhoben, die Diskussion über die Menschenrechte um eine neue Dimension. Die immer restriktivere Einwanderungspolitik Frankreichs, aber auch der anderen europäischen Länder und der Vereinigten Staaten, ging mit Polizeimaßnahmen gegenüber Immigranten einher, die der von Hannah Arendt einst angesichts der Situation Staatenloser aufgeworfenen Frage erneut Aktualität verliehen: Hängt die praktische Wirksamkeit der Menschenrechte von der Staatsbürgerschaft ab? Letzten Endes hat die Entwicklung überstaatlicher Justizbehörden - ob als ständige (Internationaler Gerichtshof [IGH], Internationaler Strafgerichtshof [IStGH]) oder vorübergehende (Internationaler Strafgerichtshof für das ehemalige Jugoslawien, Internationaler Strafgerichtshof für Ruanda [IStGHJ, IStGHR]) Einrichtungen -, die ihre Entscheidungen aus Texten herleiten, welche ihrerseits auf den Grundsätzen der Menschenrechte begründet sind, zumindest unter Juristen ein Nachdenken über Aussichten und Schwierigkeiten der Globalisierung des Rechts ausgelöst. ${ }^{9}$

Jedes dieser Elemente hat in den vergangenen dreißig Jahren zur Förderung des Nachdenkens über die Menschenrechte beigetragen, indem es den Interpretationsrahmen mehr oder weniger nachdrücklich modifizierte, in dem ihre Bedeutung analysiert wurde. Solchermaßen verlief die jüngere Geschichte der Problematisierung der Menschenrechte sukzessiv oder parallel zur Überprüfung der Beziehungen zwischen Politik und Moral, zur Debatte über den Individualismus, zur Frage staatsbürgerlichen Verhaltens oder auch zur Frage der Beziehung zwischen Universalität und Partikularität (sind die Menschenrechte eine westliche Ideologie?). Da die unterschiedlichen Etappen dieser Geschichte hier nicht rekapituliert werden können, greifen wir lediglich zwei Aspekte der französischen Debatte heraus, die die nationalen Besonderheiten der Behandlung dieser Frage von universalem Interesse erklären.

\section{Die liberale Interpretation}

6 Der erste Aspekt ist die Überdeterminierung der Deutung der Menschenrechte durch den Gegensatz, der die politisch-theoretischen Parteiungen in Frankreich - auf der einen Seite die liberalen Denker, auf der anderen eine sich mehr oder weniger auf den Marxismus berufende Linke - das ganze 20. Jahrhundert hindurch strukturierte. Gegen Ende der siebziger Jahre und mehr noch im anschließenden Jahrzehnt lieferten der Glaubwürdigkeitsverlust der revolutionären doxa, das Verblassen der theoretischen Konfiguration, die für die Verwerfung der Gestalt des Subjekts einstand, und die zweihundertste Wiederkehr des Jahrestags der Französischen Revolution den Liberalen Gelegenheit, mit Erfolg ein radikal neues Interpretationsschema der politischen Geschichte des Frankreichs dieser letzten zwei Jahrhunderte anzubieten. Die Arbeiten von François Furet, Marcel Gauchet und das 1988 (dt. 1996) veröffentlichte Kritische Wörterbuch der Französischen Revolution legten die Themen dieser liberalen Umdeutung der Französischen Revolution und ihrer Folgen fest. Selbstverständlich musste die Interpretation der Déclaration des droits de l'homme, der Erklärung der Menschenrechte von 1789, im Zentrum dieser Deutung stehen. 
7 Der Kern dieser neuen Lesart bestand in der Anprangerung des Mythos der souveränen Nation, den alle diese Autoren als vom monarchischen Zentralismus ererbtes Schema ansahen. Ihnen zufolge steht dieser Mythos am Ursprung des Legizentrismus der französischen politischen Tradition, dessen theoretisches Fundament Rousseau geliefert haben soll. Indem die Déclaration das Gesetz zum Ausdruck der volonté générale, des Willens der Allgemeinheit, erklärte (Art.6), habe sie der Forderung nach einer Beteiligung des Volks an der Ausübung der Macht den Weg gebahnt. Dass die verfassunggebende Versammlung sich trotz Rousseau der Notwendigkeit der Repräsentation beugte, hinderte diese Forderung nach Beteiligung nicht daran, in den folgenden zweihundert Jahren einem immer wiederkehrenden Protest gegen die etablierten Mächte Nahrung zu geben, auf den jene Autoren die permanente Instabilität des politischen Lebens in Frankreich zurückführen. Diese Beteiligungslogik geht ihnen zufolge einher mit einer Unterschätzung der schützenden Funktion der Menschenrechtserklärung. Gelte nämlich das Gesetz als Ausdruck des Willens der Allgemeinheit, dann gebe es keinerlei Rechtfertigung dafür, die Individuen mit Rechten auszustatten, die sie gegen ebendieses in Anspruch nehmen könnten. Der rousseauistische Legizentrismus wird daher für den »falsch verstandenen demokratischen Radikalismus ${ }^{10}$ der politischen Tradition Frankreichs verantwortlich gemacht und gleichzeitig für die Nachsichtigkeit der Linken gegenüber dem Totalitarismus der sowjetischen Regime.

Diese liberale Lesart der Déclaration von 1789 stützt sich auf einen Vergleich zwischen ihr und den amerikanischen Analoga vor dem Hintergrund einer generellen Gegenüberstellung der demokratischen Tradition Frankreichs und der Tradition Amerikas - auf einen Vergleich, der in jeder Hinsicht zugunsten der letzteren ausfällt. Im Großen und Ganzen werden die Misserfolge und Sackgassen des politischen Lebens in Frankreich dem monarchischen Erbe zugeschrieben, das der Jakobinismus lediglich neu kostümiert habe, indem er die Souveränität des Königs durch die der Nation ersetzte, und dieses Erbe habe die französische Konzeption von Demokratie bis in die letzten Jahrzehnte des 20. Jahrhunderts hinein fortwährend formatiert. Angesichts der zur Norm wohl verstandener Modernität erhobenen Amerikanischen Revolution erscheinen die Französische und die von ihr ausgehende demokratische Tradition als entschieden vergangenheitsfixiert. Als die französische Linke die lange von ihr vernachlässigten Menschenrechte wieder entdeckte, warfen die Liberalen ihr vor, dass sie der Erklärung dieser Rechte eine grundlegende Funktion für die Institutionen beimesse und ihre Forderung nach Beteiligung des Staatsbürgers auf sie gründe. Was die Linke hartnäckig übersehe, sei die erforderliche Trennung zwischen privater und öffentlicher Sphäre und damit die "Differenz des Politischen ${ }^{11}$, das heißt die Außenstellung der Macht gegenüber der Gesellschaft. Dem gegenüber hielten die Liberalen auf die politische Bedeutung der Menschenrechte in ihrer amerikanischen Interpretation, die darin bestehe, dass diese nicht dazu bestimmt seien, Macht $\mathrm{zu}$ begründen, sondern sie $\mathrm{zu}$ begrenzen, die Individuen mithin gegen Regierungsinstanzen zu schützen, die zwar notwendig, aber von der Gesellschaft geschieden und weiterhin zu scheiden seien. Die amerikanischen Revolutionäre hätten die virtuelle Repressivität der Macht keineswegs verkannt. Im Unterschied zu den Franzosen hätten sie sich jedoch nicht dadurch gegen sie zu verwahren gesucht, dass sie den Unterschied zwischen Macht und Gesellschaft verwischten, sondern indem sie die Institutionen derart gestalteten, dass unterschiedliche Machtorgane sich gegenseitig begrenzen und einschränken. Eher als Rousseau habe Montesquieu sie 
inspiriert, und in einem ausgeklügelten, von pragmatischen Betrachtungen ausgehenden Aufbau der Institutionen hätten sie eher eine sicherere Gewähr der Menschenrechte erblickt als in der Erhebung des Volks zum Gesetzgeber. ${ }^{12}$

\section{Die neuen Lesarten der Linken}

9 Die liberale Deutung hat Epoche gemacht und findet auch heute noch Anhänger. Aber die Dissidenten des Ostblocks, der Zusammenbruch des Sowjetsystems und das zweihundertjährige Bestehen der Déclaration des droits de l'homme haben bei Theoretikern der Linken ebenfalls neue Überlegungen ausgelöst. Zwar fanden diese Überlegungen in den Medien weniger Anklang als die der Liberalen, aber sie eröffneten doch auch ihrerseits ungewöhnliche Perspektiven. Die Texte von Claude Lefort und Étienne Balibar (beide hier in deutscher Übersetzung) sind gute Beispiele dafür. Sie sprechen für sich, und es dürfte überflüssig sein, sie vollständig zu resümieren. Wir begnügen uns damit, die Gründe aufzuzeigen, derentwegen sie sich heute, zwanzig beziehungsweise dreißig Jahre nach ihrer Erstveröffentlichung, unserer Aufmerksamkeit empfehlen.

10 Nach dem Muster der Liberalen leitet Lefort seine Interpretation der Menschenrechte von der Kritik des Totalitarismus her, und wie sie reagiert er darauf, dass Linke und Linksradikale vom Bezug auf die Menschenrechte in der zweiten Hälfte der siebziger Jahre kompensatorischen Gebrauch machten. Aber trotz seiner Distanz zu Marx und mehr noch zum Marxismus der Kommunistischen Partei oder der radikalen Linken stellt er seine Analysen unter das Vorzeichen einer als Emanzipationsprozess verstandenen demokratischen Logik. Bemerkenswerterweise fehlt bei ihm der Gegensatz zwischen französischer und amerikanischer Tradition. Es geht ihm nämlich nicht darum, eine orthodoxe Position hinsichtlich des richtigen Aufbaus der Institutionen festzulegen, sondern um den radikal innovatorischen Charakter des Prinzips der Déclaration selbst. Dass überhaupt Menschenrechte formuliert wurden, ist ihm wichtiger als ihr Inhalt. Durch die Tatsache ihrer Formulierung seien die von den Menschen in Gesellschaft wahrgenommenen Rechte nämlich nicht mehr wie zuvor auf eine unwandelbare Sozialordnung oder auf einen Vertrag zwischen der Macht und ihren Untergebenen bezogen, sondern eben auf den Akt ihrer Formulierung. Wenn Lefort wie die Liberalen den von der Französischen Revolution vollzogenen Bruch relativiert und eine gewisse Kontinuität zwischen ihr und der vorangegangenen monarchischen Zentralisierung anerkennt, situiert er diese Kontinuität doch nicht auf symbolischer Ebene, im Mythos der souveränen Nation, sondern in den administrativen Strukturen der gesetzlich ausgeübten Macht über ein Territorium: Die Revolution habe nicht den Rechtsstaat errichtet, sie habe jedoch innerhalb eines bereits bestehenden Rechtsstaats die Beziehungen zwischen Macht und Recht tiefgreifend verändert. Lefort zufolge war die Déclaration des droits de l'homme das entscheidende Instrument dieses Wandels. Gegen die Marxsche Interpretation vertritt er die Auffassung, der von den Menschenrechten vorausgesetzte Mensch sei nicht der Eigentümer, das heißt das egoistische Individuum der bürgerlichen Gesellschaft; freilich auch nicht das Individuum, das den Unterschied zwischen Privatsphäre und Öffentlichkeit verkennt und Freiheit mit Beteiligung an der Macht einer größeren Gruppe verwechselt. Das auf die unbestimmte Entität des »Menschen« im Allgemeinen bezogene Recht konstituiere eine der Macht unaufhebbar entzogene, 
»undomestizierbare Quelle von Legitimität « ${ }^{13}$, die die Möglichkeit impliziere, die gegebene Ordnung jederzeit in Frage zu stellen. Es breche damit einer Geschichte Bahn, in der »die erworbenen Rechte notwendig aufgefordert sind, neue Rechte $\mathrm{zu}$ unterstützen «. ${ }^{14}$

In einer etwas anderen Perspektive als Lefort, die aber in einem wesentlichen Punkt zu konvergierenden Schlussfolgerungen führt, wendet sich auch Balibar gegen die Marxsche Lesart der Déclaration von 1789. Marx' Irrtum bestand für Balibar darin, dass er das liberale Verständnis des Begriffspaars homme-citoyen, Mensch-Staatsbürger, übernahm, indem er es als Gegensatz interpretierte. Gerade in der Gleichsetzung der beiden Begriffe bestehe jedoch die große Innovation jenes Textes, das, was seinen revolutionären Charakter ausmache. Weit davon entfernt, bloß die dem Menschen als Privatindividuum gewährten Rechte darzustellen, seien die Menschenrechte politisch im vollen Sinn des Wortes. Genauer: Sie bekundeten die Idee eines Rechts der Menschen, aller Menschen, omnes et singulatim, auf Politik. Diese Gleichsetzung setze eine weitere voraus, die Balibar in einer Wortschöpfung zusammenfasst: Égaliberté, "Gleichfreiheit«. Entgegen der Auffassung, die im 19. und 20. Jahrhundert zu einem Axiom des Liberalismus werden sollte (einem vom Sozialismus, der aus ihm freilich ganz andere Folgerungen zog, beglaubigten Axiom), habe die Logik der Déclaration Freiheit und Gleichheit ebenso wenig als Gegensatz behandelt wie sie den Menschen vom Staatsbürger unterschied. Da sie vielmehr Freiheit und Gleichheit als koextensiv gesetzt habe, habe sie einen Prozess der Politisierung der Forderung nach Rechten in Gang gebracht, in deren Dynamik sich alle Emanzipationskämpfe der beiden folgenden Jahrhunderte eingereiht hätten. Wenn Balibar Marx nachspricht, dass »die Emanzipation der Unterdrückten nur ihr eigenes Werk sein kann«, schließt er sich zugleich Leforts Idee einer »undomestizierbaren Quelle von Legitimität« in den modernen demokratischen Systemen an. Beide Autoren unterstreichen, dass dieses Prinzip eine strukturelle Instabilität jener Systeme zur Folge habe, eine Instabilität, die zu unterdrücken jedoch nicht nur illusorisch, sondern auch gefährlich wäre insofern, als sie gerade aus dem resultiere, was deren eigentlich demokratisches Moment darstelle. Weiterhin geht Balibar den Widersprüchen nach, die dem postrevolutionären Begriff des Politischen - teils Erfordernis der Institution, teils Legitimation des Protests gegen jede ihrer erstarrten Formen (oder in seinen Worten: teils Logik der Verfassung, teils Logik des Aufstands) - inhärent seien, und entwickelt die Aporien einer tendenziell universalen Staatsbürgerschaft, die sich anscheinend doch nur in institutionellen Formen verkörpern könne, die die Exklusion verlagern, ohne sie je abschaffen zu können.

12 Einige Worte $\mathrm{zu}$ meinem eigenen Text und dem Zusammenhang, in dem er zu den beiden vorangegangenen steht. Er unterscheidet sich von ihnen durch seinen Ausgangspunkt, der nicht die Interpretation der Déclaration des droits de l'homme ist, sondern ein Versuch, den von Michel Villey und schon viel früher von einigen Rechtstheoretikern untersagten Begriff des "subjektiven Rechts « zu rehabilitieren. ${ }^{15} \mathrm{Er}$ trifft sich mit jenen Texten jedoch darin - und hier zeigt sich, wie sehr die Bedeutung der Menschenrechte an die Originalität der juristischen Figur des Rechtssubjekts gebunden ist -, dass er die Historizität des modernen politischen Subjekts wie auch die damit im Zusammenhang stehende Historizität der Demokratie unterstreicht. Deren Wirklichkeit beruht nicht auf den Strukturen einer Regierungsform (obwohl diese Strukturen ihr nicht ganz äußerlich sind), sondern auf der Dynamik ihrer stets erneuerten Infragestellung und der aus dieser Infragestellung resultierenden 
institutionellen Transformationen. Anders gesagt: Motor der Demokratie sind die Kämpfe, die von den unter welchem Vorwand auch immer (als Arbeiter, Frauen, Schwarze, Immigranten usw.) von der Macht Ausgeschlossenen im Namen der Gleichheit gegen die etablierten Mächte geführt werden. Diese Proposition greift nur auf, was bereits Lefort, Balibar und übrigens auch Rancière ${ }^{16}$ thematisiert haben. Sie mag als Ausdruck einer "radikaldemokratischen" Einstellung angesehen werden. Aber die vor zwei Jahrzehnten vollzogene Verlagerung von der Frage der Revolution zu der der Staatsbürgerschaft hatte zur Folge, dass dieser Radikalismus nicht mehr auf die Austilgung der Macht zielt, auch nicht auf deren Eroberung oder die Beteiligung an ihr, sondern dass sie ihre unreduzierbare Äußerlichkeit zur Kenntnis nimmt. Die Macht wird nunmehr verstanden als notwendiger Partner innerhalb einer konfliktuellen Beziehung, die nur vorübergehende Lösungen erfahren kann, als Adressat von Forderungen, die ihre Existenz voraussetzen und zugleich unablässig in Frage stellen.

\section{Menschenrechte, subjektive Rechte: ihre Zukunft}

Angesichts dessen ist es nicht verwunderlich, dass das Nachdenken über die Menschenrechte, das die Kommentare zu den revolutionären Menschenrechtserklärungen ganz natürlich mit dem Verständnis der Staatsbürgerschaft verbunden hatten, in den letzten Jahrzehnten auf die Analyse zeitgenössischer Figuren der Exklusion stieß: der Obdachlosen, der Arbeitslosen und auch der Immigranten ohne Aufenthaltsgenehmigung. Das ist der zweite Aspekt der zeitgenössischen französischen Debatte über die Menschenrechte; in den hier übersetzten Texten zeichnet er sich bereits ab. Unter all den »Anteillosen« (den sans part, wie Rancière sie nennt), deren Aktionen zur Erlangung neuer Rechte unser Verständnis von Staatsbürgerschaft neu definieren helfen, ist der Immigrant heute wohl die repräsentativste Gestalt. ${ }^{17}$ Die Schaffung einer europäischen Staatsbürgerschaft durch den Vertrag von Maastricht (1992) hat das Band gegenseitiger Implikation von Staatsbürgerschaft und Staatsangehörigkeit gelockert, das nahezu zwei Jahrhunderte lang unser staatsbürgerliches Verständnis bestimmt hatte. Da aber diese europäische Staatsbürgerschaft oft bloß als Reproduktion des Modells nationaler Staatsbürgerschaft auf erweiterter Stufenleiter gedacht wird, integriert auch sie nur, indem sie zugleich ausschließt. Das Schicksal der Staatenlosen in der Zwischenkriegszeit und während des Zweiten Weltkriegs hatte Hannah Arendt dazu veranlasst, den Begriff eines »Rechts auf Rechte« zu formulieren, das heißt eines Rechts auf politische Inklusion, anscheinend die Voraussetzung dafür, dass die Menschenrechte auch praktisch gelten. In unseren Tagen verleiht die Lage des Asylantragstellers oder des immigrierten Arbeiters ohne Aufenthaltsgenehmigung diesem Begriff neue Aktualität und veranlasst dazu, hierin den radikalsten, aber auch konsequentesten Ausdruck des Staatsbürgerbegriffs der revolutionären und postrevolutionären Epoche zu erblicken.

Die liberalen Lesarten der Déclaration des droits de l'homme wollten ein Kapitel der Geschichte abschließen, das der Revolutionen. Die Lesarten der Linken hingegen unterstreichen die emanzipatorische Bedeutung der Forderung nach Rechten, die sich nicht auf die Vergangenheit berufen, sondern auf eine Natur, der einzig erworbene und im allgemeinen schwer erkämpfte - Rechte eine Bestimmtheit verleihen können, die ihr ursprünglich fehlt, und bereiten damit der Zukunft den Weg. Die demokratische 
Revolution ist nie vollendet, weil der Sinn der Demokratie sich nicht in institutionellen Arrangements erschöpfen kann. Gewiss, einige sind anderen vorzuziehen, vielleicht unter dem Gesichtspunkt der Stabilität (aber es gibt auch Diktaturen, die langlebig sind, was nicht genügt sie zu rechtfertigen), sicherlich unter dem Gesichtspunkt der Möglichkeiten, die sie einer Opposition bieten - aber keine von ihnen leistet definitiv Gewähr gegen ein Abdriften der Macht. Die Vitalität der Demokratie, ihre Fähigkeit, unvorhersehbare soziale und kulturelle Veränderungen zu integrieren, hängt indes mindestens ebenso von den Forderungen der Ausgeschlossenen ab wie von der guten oder schlechten Politikgestaltung der Machthaber. ${ }^{18}$ Einer der Vorteile eines stärker an staatsbürgerlichen Praktiken als an Formen der politischen Systeme ausgerichteten Demokratieverständnisses liegt darin, dass es der Staatsbürgerschaft andere Räume eröffnet als nationale. Wohl aufgrund des Beharrungsvermögens der republikanischen Tradition Frankreichs, für die der Nationalstaat den verbindlichen Rahmen der "Gemeinschaft der Staatsbürger « darstellt, ${ }^{19}$ haben die französischen Philosophen sich insgesamt bisher wenig für den Aufbau Europas interessiert, und weniger noch für die politischen, juristischen und gerichtlichen Dimensionen der Globalisierung. Die Brücke zwischen ihrem Denken und den von Juristen und Politikexperten angestellten Überlegungen bleibt zu schlagen. Ich tendiere zu der Auffassung - aber ich spreche dabei nur für mich -, dass genau diese Brücke es ermöglichen wird, alle in der Figur des Staatsbürgers als Rechtssubjekt enthaltenen Ressourcen zu entfalten und damit weitere Schritte auf dem Weg zu der bereits angebahnten Dissoziierung von Staatsbürgerschaft und Staatsangehörigkeit zu unternehmen.

\section{BIBLIOGRAPHIE}

Badie, B. (2002): La diplomatie des droits de l'homme, Paris, Fayard.

Balibar, E. (1989): »La proposition de l'égaliberté«, in: Les conférences du Perroquet, 22.

Balibar, E. (2002): Droits de cité, Paris, PUF.

Balibar, E. (2002-2003): »Nouvelles réflexions sur l'égaliberté« (unveröff. Ms.).

Binoche, B. (1989): Critiques des droits de l'homme, Paris, PUF.

Binoche, B. / Cléro, J.P. (2007): Bentham contre les droits de l'homme, Paris, PUF.

Bourgeois, B. (1990): Philosophie et droits de l'homme, Paris, PUF.

Delmas-Marty, M. (Hg.) (1989): Raisonner la raison d'Etat. Vers une Europe des droits de l'homme, Paris, PUF.

Delmas-Marty, M. (2004): Le relatif et l'universel, Paris, Seuil.

Delmas-Marty, M. (2006): Le pluralisme ordonné, Paris, Seuil.

Delmas-Marty, M. (2007): La refondation des pouvoirs, Paris, Seuil.

Descombes, V. (2004): Le complément de sujet, Paris, Gallimard. 
Gauchet, M. (1980): »Les droits de l'homme ne sont pas une politique«, in: Le Débat, 3, S. 3-21.

Gauchet, M. (1996 [1988]): »Menschenrechte«, aus dem Französischen von Christian Voigt, in: Furet, F. / Ozouf, M. (Hg.): Kritisches Wörterbuch der Französischen Revolution, Frankfurt a.M., Suhrkamp, S. 1180-1198.

Gauchet, M. (1989): La Révolution des droits de l'homme, Paris, Gallimard.

Kervégan, J.-F. (1995): »Les droits de l'homme«, in: Kambouchner, D. (Hg.): Notions de Philosophie, Bd. II, Paris, Gallimard, S. 637-696.

Lefort, C. (1994), „Droits de l'homme et politique«, in: ders.: L'invention démocratique, Paris, Le livre de poche.

Lefort, C. (2007): »La pensée politique devant les droits de l'homme«, in: ders.: Le temps présent, Paris, Belin.

Lochak, D. (2002): Les droits de l'homme, Paris, La Découverte.

Lochak, D. (2007): Face aux migrants: Etat de droit ou état de siège?, Paris, Textuel.

Rancière, J. (2004): Aux bords du politique, Paris, Gallimard.

Sassen, S. (2008 [2006]): Das Paradox des Nationalen. Territorium, Autorität und Rechte im globalen Zeitalter, aus dem Amerikanischen von Nikolaus Gramm, Frankfurt a.M. Suhrkamp.

Schnapper, D. (1994): La communauté des citoyens, Paris, Gallimard.

Villey, M. (1983): Le droit et les droits de l'homme, Paris, PUF.

Villey, M. (2003): La formation de la pensée juridique moderne, Paris, PUF.

\section{NOTES}

1. Villey (1983).

2. Vgl. Descombes (2004).

3. Gauchet (1980).

4. Binoche (1989).

5. Lefort $(2007 ; 1994)$.

6. Gauchet (1988); Balibar (1989).

7. Bourgeois (1990).

8. Badie (2002).

9. Delmas-Marty $(2004 ; 2006 ; 2007)$.

10. Gauchet (1989), S. 85.

11. Gauchet (1989), S. 79.

12. Für eine nuanciertere Interpretation des Unterschieds zwischen der französischen und der amerikanischen Menschenrechtserklärung vgl. Kervégan (1995).

13. Lefort (2007), S. 64.

14. Lefort (2007), S. 67.

15. Es gibt in Frankreich durchaus Arbeiten von Juristen und Rechtshistorikern zum Begriff der subjektiven Rechte. Auf Seiten der politischen Philosophie jedoch haben sich die Überlegungen auf die Frage der Menschenrechte und ihre Beziehung zu den staatsbürgerlichen Rechten fokussiert. Die historische Originalität der Denkfigur subjektives Recht und ihre Implikationen bezüglich der Modalitäten sozialer Inklusion sind wenig erörtert worden.

16. Vgl. Rancière (2004). 
17. Vgl. Balibar (2002); hier vor allem die Texte »Ce que nous devons aux ১Sans-Papiers« und »Une citoyenneté européenne est-elle possible?«.

18. Vgl. dazu auch Sassen (2008 [2006]), S. 463-466. Im Rahmen ihrer Überlegungen zur Komplexität der Staatsbürgerschaft heute und zur Entstehung von Rechtssubjekten entwickelt Saskia Sassen die These, der zufolge »die Staatsbürgerschaft teilweise durch die Praktiken der Ausgeschlossenen konstituiert wird«, die das Feld eröffnen für »die Rechte nicht formalisierter Akteure und Fragestellungen, insbesondere wenn die Hoheit des Nationalstaates über die Themenbereiche von Identität und Zugehörigkeit aufgrund wichtiger gesellschaftlicher, wirtschaftlicher, politischer und subjektiver Trends nachgelassen hat« (S. 463).

19. Vgl. Schnapper (1994).

\section{AUTEURS}

\section{CATHERINE COLLIOT-THÉLÈNE}

Catherine Colliot-Thélène ist Professorin für Philosophie an der Universität Rennes I. Nähere Informationen finden Sie hier. 